\title{
ALEXA CONTROLLED HOME AUTOMATION SYSTEM
}

Ujjwal Mathur', Swapnil Kumar Yadav', Tushar Tevetia', Geetika Aswani ${ }^{4}$

E-Mail Id: ${ }^{1}$ ujjwal.16ben1015@abes.ac.in, ${ }^{2}$ swapnil.16ben1066@abes.ac.in, ${ }^{3}$ tushar.16ben1031@abes.ac.in, ${ }^{4}$ geetika.aswani@abes.ac.in

ABES Engineering College, Ghaziabad, Uttar Pradesh, India

\begin{abstract}
Dynamic In the current day world IOT based keen home execution has become a genuine enticement and has additionally become out to be a significant region of examination. This exploration propounds a methodology for keen home computerization by means of IOT that is remotely controllable. An IOT based home computerization framework is required to screen and control home apparatuses remotely over the web. In this framework mobiles and PCs are utilized for controlling the essential home apparatus and make it work through the structured pages or versatile applications with neighborhood) servers or web association, therefore the house is known as a brilliant home. This idea of steering computerization in the lodging part is spreading like fire in the bramble. Western societies have greeted the idea of home robotization wholeheartedly. INDIA is additionally keeping a similar pace of modernisation as western nations seem to be. Energetic home mechanizing approaches are used, and IOT demonstrated as plan to the star among them. It gives plausibility to get to the home robotization framework from anyplace round the globe utilizing web associations. It limits superfluous human endeavors and builds up the way of life of the nation's kin.
\end{abstract}

Keywords: IOT, Home robotization.

\section{INTRODUCTION}

The worldwide interest of vitality utilization has reached nearly to its zenith. With the development of condition of craftsmanship innovations, the interest for power as a wellspring of vitality has likewise expanded. Vitality in its root structure is used from a pencil to a hydroelectric venture with the development popular the movement of vitality utilization is likewise developing. A large number of dollars are spent by enterprises and mechanical units not exclusively to discover new wellsprings of vitality yet additionally on the approaches to monitor it.

Home computerization frameworks run on the possibility of less utilization of mechanical and electrical vitality, eventually diminishing human endeavors by welcoming procedure on our fingertips and expanding investment funds in the method of cost effective bills

Amazon Echo is a voice empowered remote speaker which is created by amazon itself. The gadget can interface with the voice-controlled insightful individual colleague administration Alexa, which reacts to the name "Alexa". This gadget isn't just equipped for voice association yet can likewise do music playback, making daily agendas, setting alerts, spilling web recordings, playing book recordings, and giving climate, traffic and other constant information. Being a home computerization center it can likewise control a few savvy gadgets. Home computerization is an exorbitant utility that numerous individuals in India and different nations can't manage. The target of this item is to give a modest and simple approach to control non-brilliant gadgets utilizing the intensity of voice. We utilize the Amazon reverberation to build up an ability (application) that will speak with our ESP8266 NodeMCU controller to control our gadgets. At whatever point we need to turn on or turn off apparatuses associated with the equipment, we ask the Amazon Echo and it would do the needful.

\subsection{Design Considerations}

The most significant part of home computerization is client accommodation. It tends to be utilized to spare vitality and develop client ease.it is notable that there are huge number of home robotization framework accessible however they fluctuate as far as dependability, moderateness and in particular to withstand immensely significant trial of time

\subsubsection{Client Order}

The client's info is alluded to as client order, it means the activity that a specific individual needs the framework to perform. For instance - turning on or turning off the home lights, controlling the cooling framework, opening the primary entryway and so forth.

\subsubsection{IoT Based Server}

The client's order comes to the microcontroller of the web of things, where the microcontroller is associated with the LAN which is at last associated with the web. In this way the order is transmitted from the microcontroller to the LAN IOT Based Server-the client's order comes to the microcontroller of the web of things, where the microcontroller is associated with the LAN which is at last associated with the web. In this way the order is transmitted from the microcontroller to the LAN

\subsubsection{Microcontroller Command Line}

Command is received by the microcontroller and the activity is at last executed by the framework. 
ICACCG2020 30-31 July, 2020, Ansal University, Gurgaon, India

International Journal of Technical Research \& Science (Special Issue)

ISSN No.:2454-2024 (online)

\subsection{Problem Statement}

A home mechanization framework is structured utilizing IOT which empowers us to robotize fundamental home functionalities like turning off/on types of gear, cooling frameworks, fridges and other home operational apparatuses.

\section{TECHNOLOGY STACK}

In this undertaking, numerous specialized segments are incorporated and a consistent usefulness among them is built up. Our specialized stack is outlined as follows

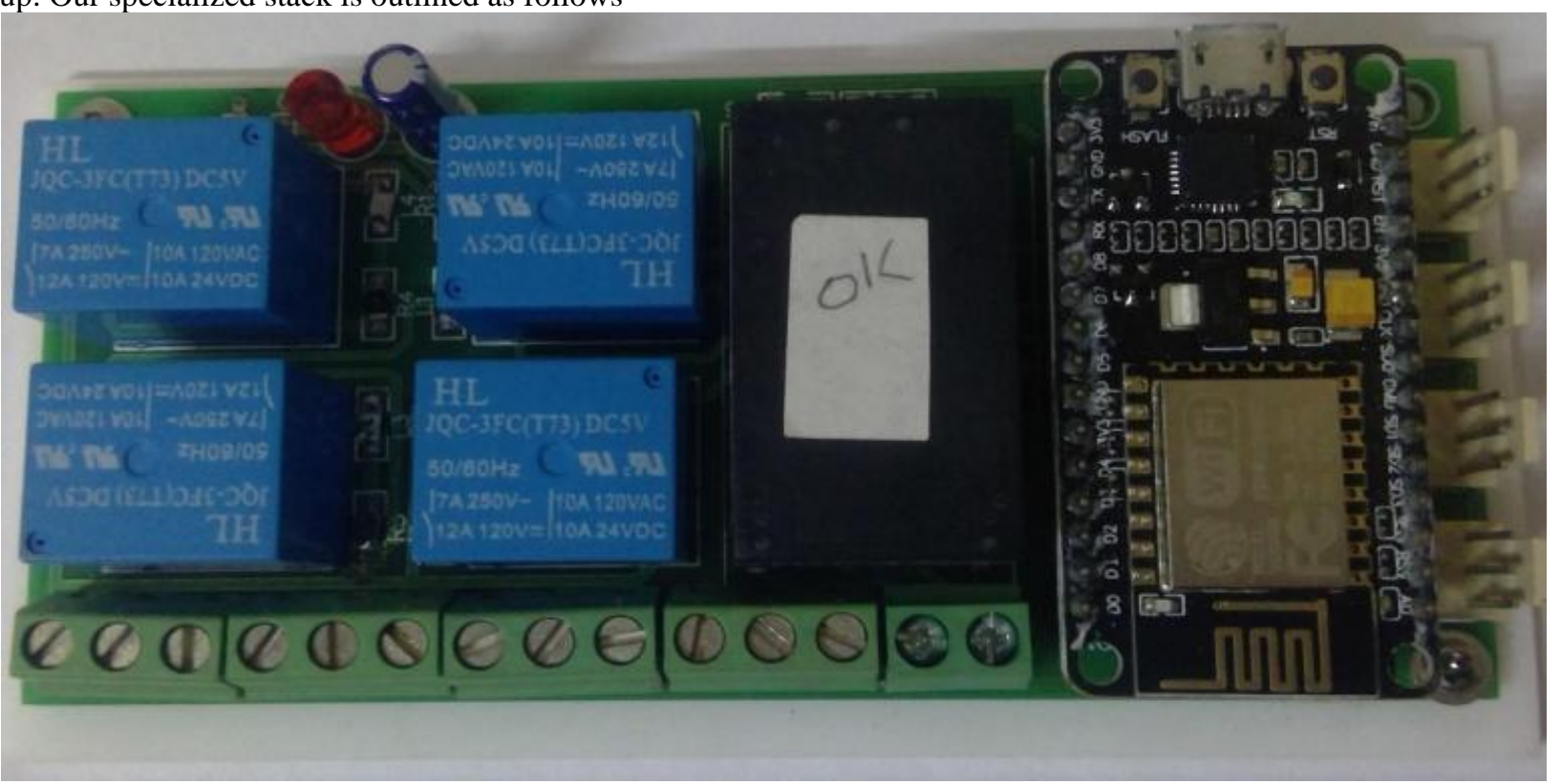

Fig. 2.1 Hardware PCB

\subsection{Physical Layer}

This comprises of a layer with which a client connects:

\subsubsection{Node MCU}

It is an open-source firmware and advancement pack which will help you in prototyping or building IoT items. It incorporates firmware that suddenly spikes in demand.

\subsubsection{Amazon Dot}

Shrewd Personal Assistant gadget which is available in client's house which is activated utilizing vocal orders. It reacts based on client's solicitation being made

\subsection{Program Layer}

The programming of microcontroller is done using Embedded $\mathrm{C}$ language which is specialized banter is recorded as under:

\subsubsection{Devices}

The data sources are given by means of Amazon's reverberation. Reverberation's regular exact voices result from discourse unit determination innovation. It can see what the clients are stating utilizing NLP calculations incorporated with the Echo's content to-discourse (TTS) motor. The Echo equipment supplement incorporates a Texas Instruments DM3725 ARM Cortex-A8 processor, 256MB of LPDDR1 RAM and 4GB of extra room. It associates with the web through Wi-Fi $802.11 \mathrm{a} / \mathrm{b} / \mathrm{g} / \mathrm{n}$.

\subsubsection{Framework}

Alexa voice administrations powers Amazon Echo by changing over discourse into text and giving astute answers to client demands. Alexa Dot is equipped for vocal connection, playing music, scheduling plans for the day, setting alerts, spilling digital broadcasts, playing book recordings, and giving climate, traffic and other ongoing data. Alexa can likewise control a few brilliant gadgets utilizing itself as a home computerization center point.

\section{SYSTEM ARCHITECTURE}

\subsection{Design Overview}

Our framework shown in Fig. 3.1-PCB, NodeMCU and the full equipment which is utilized in controlling exchanging activity. This segment clarifies quickly about the job of every segment to make the framework 
ICACCG2020 30-31 July, 2020, Ansal University, Gurgaon, India

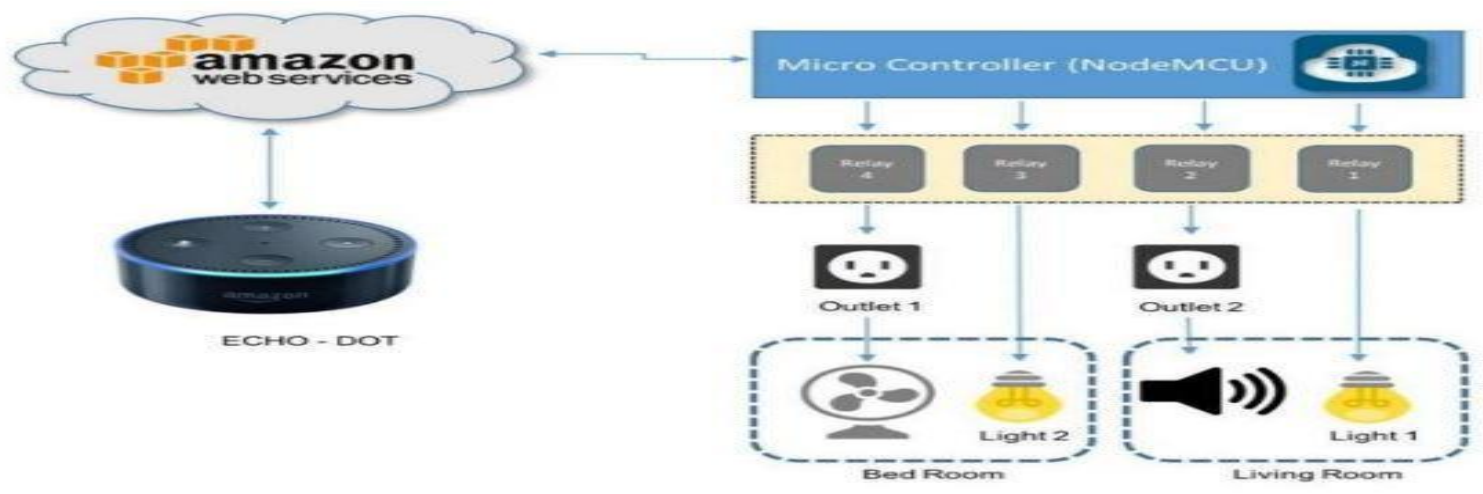

Fig. 3.1 System Overview

\subsection{Amazon Dot}

Alexa Voice Service is the insightful vocal control administration which controls gadget, Amazon Echo. Alexa utilizes characteristic language handling procedures prepared by the designers and the client International Journal of Current Engineering and Scientific Research (IJCESR) ISSN (Print): 2393-8374, (Online): 2394-0697, Volume-5, Issue-4, 201851 network of Amazon to process client asks for and take into account their needs. The vocal administration can be activated utilizing the catchphrase "Alexa". As referenced before, the expertise/application that we have created can be activated utilizing the voice order, "Alexa, Turn the lights on". On activating of alexa,a content is sent to the cloud which understudies send the subordinate to the NodeMCU to turn the light on once the accumulation is finished by the controller, it sends back the affirmation to Alexa . what's more, the significant data is passed back to the client

\subsection{Node MCU}

The application depends over NodeMCU as it aggregates both equipment necessities and furthermore does all the calculations. The NodeMCU has an inbuilt Wi-Fi highlight and it utilizes the hand-off to turn on/off the machines

\section{CONCLUSION}

It is ordinarily knowledgeable that the web of things is the most widely recognized element utilized in home robotization frameworks. It makes living simple and is likewise useful to distinctively abled people. This task manages the way to deal with create web of things (iot) based home robotization frameworks. In this the controller board as an arduino ESP8266 with remote modules prepared is utilized to control the framework. A HTML page is likewise intended for controlling the gadgets. For the product reason implanted $\mathrm{C}$ is utilized. The primary central purposes of this task are the wellbeing and security point of view of home mechanization which are controllable and computerized in a savvy home from anyplace round the server frameworks. It spares vitality as it is exceptionally structured such that empowers cripple individuals to keep a control on their home apparatuses through their cell phones. Due to being associated through Wi-Fi it empowers to control the framework from 7 to 8 gadgets one after another which was unrealistic utilizing Bluetooth and the range was additionally brought together locally. It limits pointless human endeavors and furthermore builds up the way of life of the individuals in the general public which thus encourages us to make our carries on with a lot less complex and agreeable.

\section{REFERENCES}

[1] K. Venkatesan and Dr. U. Ramachandraiah, "Arranged Switching and Polymorphing Control of Electrical Loads with Web and Wireless Sensor Network", 2015 International Conference on Robotics, Automation, Control and Embedded Systems (RACE), Chennai, (2015), 1-9

[2] Raj, J. S. (2019). "QoS streamlining of vitality proficient steering in iot remote sensor systems'. Diary of ISMAC, $1(01), 12-23$.

[3] Vishwateja Mudiam Reddy, Naresh Vinay, Tappan Pokharna and Shashank Shiva Kumar Jha, 'Web of Things Enabled Smart Switch', Thirteenth International Conference on Wireless and Optical Communications Networks (WOCN), Hyderabad, (2016),1-4

[4] https://cityos-air.readme.io/docs/esp8266-nodemcu

[5] Kabita Agarwal and Arun Agarwal, "Audit and Performance Analysis on Wireless Smart Home and Home Automation utilizing IoT", Proceedings of the Third International Conference on I-SMAC (IoT in Social, Mobile, Analytics and Cloud) (I-SMAC 2019).

[6] T.S. Karthik and K. Malini, "VOICE BASED HOME AUTOMATION USING AMAZON DOT", 2018 INTERNATIONAL JOURNAL OF CURRENT ENGINEERING AND SCIENTIFIC RESEARCH (IJCESR).

[7] Sneha Matriaya and Dr. Ravindra Purwar, "Voice Controlled Home Automation Using Amazon Echo Dot", IOSR Journal of Computer Engineering (IOSR-JCE), Volume 20, Issue 3, Ver. III (May. - June. 2018), PP 1619. 